\title{
Maintenance Metronomic Chemotherapy in Treatment of Metastatic Colorectal Cancer
}

\author{
O. V. Streltsova ${ }^{1}$, A. V. Prokharau ${ }^{1}$, A. S. Portyanko ${ }^{2}$, E. I. Suboch ${ }^{2}$, K. N. Batura ${ }^{2} \&$ Y. V. Baranau ${ }^{3}$ \\ ${ }^{1}$ Belarusian State Medical University, Minsk, Belarus \\ ${ }^{2}$ N.N. Alexandrov National Cancer Center of Belarus, Minsk, Belarus \\ ${ }^{3}$ Minsk City Clinical Oncological Dispensary, Minsk, Belarus \\ Correspondence: A. V. Prokharau, Head of Department of Oncology, Belarusian State Medical University, \\ Dzerzhinski ave., 83, Minsk, 220116, Belarus. E-mail: aprokharau@gmail.com
}

Received: May 29, 2019

doi:10.5539/cco.v8n1p35
Accepted: June 14, 2019 Online Published: June 28, 2019

URL: https://doi.org/10.5539/cco.v8n1p35

\begin{abstract}
Treatment of metastatic colorectal cancer (mCRC) is one of the most challenging and important problems in oncology at present moment. This article presents the interim results of treatment of patients with colorectal cancer, who were enrolled from 2016 till $2019(n=60)$ with the use of maintenance metronomic chemotherapy. Metronomic regimen consisted of oral capecitabine $500 \mathrm{mg} 3$ times a day and oral cyclophosphane $50 \mathrm{mg}$ daily. The control arm consisted of mCRC patients who received the same induction chemotherapy without maintenance from 2011 till 2015 ( $\mathrm{n}=70)$. Median follow-up time was 18.5 months. Median progression-free survival (PFS) was 9.0 and 7.4 months in the maintenance and control arms respectively. Median overall survival (OS), counted from the beginning of induction chemotherapy, is currently 22.9 months in the maintenance arm, and 14.7 months in control. High expression levels of genes, encoding enzymes TS (thymidylate synthetase), DPD (dihydropyrimidine dehydrogenase) and receptor VEGFR1, low expression level of gene TP (thymidylate phosphorylase), as well as low levels of tumor markers CEA and CA 19-9 are the prognostic factors of sensitivity to metronomic chemotherapy given to colorectal cancer patients. Based on these data, we identified a group of patients who are recommended to use this method of treatment.
\end{abstract}

Keywords: metronomic chemotherapy, angiogenesis, survival rates, colorectal cancer, maintenance therapy

\section{Introduction}

Colorectal cancer is now on the third place among oncological diseases for its frequency both in the Republic of Belarus and in the whole world and is one of the main causes of cancer deaths. Five-year survival of patients with distant metastases not eligible for surgical treatment is less than 5\% (Ferlay, Steliarova-Foucher \& Lortet-Tieulent, 2012; Okeanov, Moiseyev \& Levin, 2014). The improvement of efficacy of colorectal cancer treatment is one of the urgent problems of modern oncology because of incidence rate growth, high metastasis frequency and low survival rates.

Chemotherapy plays a major role in treatment of patients with mCRC. It has made significant progress during the last years: with modern chemotherapy regimens life expectancy exceeds 20 months. However, up to now the question of therapy duration remains completely unsolved. One of the probable treatment strategies is so called maintenance treatment, which means that after the completion of standard induction therapy, a patient continues to take less toxic drugs until tumor progression (Chibaudel et al., 2012).

Metronomic chemotherapy is one of the promising methods of maintenance treatment of different kinds of tumors. During such therapy cytostatic drugs are administered at relatively low, minimally toxic doses without long intervals between administrations. In this case, primarily not the cytostatic but the antiangionic effect of drugs is realized, as well as their ability to affect the tumor cell microenvironment, to exert an immune response. (Browder, Butterfield, \& Kraling, 2000). The main target of metronomic chemotherapy is the endothelium of tumor-associated vessels. All known cytostatic drugs can find use in metronomic regimens; however more often cyclophosphane, methotrexate and capecitabine are used (Gnoni, Silvestris, \& Licchetta, 2015).

Nowadays metronomic chemotherapy is more commonly used to treat recurrent forms of tumors refractory to other methods. Several clinical trials studied the effectiveness of capecitabine in metronomic regimen and a 
combination of capecitabine and cyclophosphane at low doses for colorectal cancer patients after progression on standard chemotherapy, as well as for elderly patients with intolerance of other treatments (Miger, Holmqvist, Sun, \& Albertsson, 2012; Allegrini, Di Desidero, \& Barletta, 2012). Capecitabine dose varied from 500 to 2000 $\mathrm{mg}$ a day and cyclophosphane dose usually was $50 \mathrm{mg}$ a day. Moderate efficacy and low toxicity of used methods was proved. The present study aims to evaluate the role of metronomic regimen including low doses of capecitabine and cyclophosphane in maintenance treatment of mCRC patients who have received the first-line induction chemotherapy. We also studied the influence of different clinical and molecular genetic factors on the efficacy of metronomic chemotherapy.

Among the most studied markers of sensitivity to fluoropyrimidine, one of which is capecitabine, are expression levels of genes, encoding enzymes TS, TP and DPD (Longey, Harkin, \& Johnston, 2003; Stohmacher, 2005). Also, a potential marker of sensitivity to metronomic chemotherapy is the vascular endothelium growth factor (VEGF) (Kerbel, 2004). According to the data of several studies in small groups, it was established that patients with low VEGF level and those with its decrease on treatment had higher survival rates (Calleri, Bono, Bagnardi, \& Quarna, 2009; Drevs, Fakler, \& Eisele, 2004). Thus, the VEGF level in blood serum and also the expression of its receptors VEGFR1(Flt-1) and VEGFR2(KDR) in the tumor tissue are the potential biomarkers of sensitivity to metronomic chemotherapy regimens that require a further research in large patient groups.

\section{Materials and Methods}

\subsection{Patients}

The present study is an interim analysis of treatment of mCRC patients, who received maintenance metronomic chemotherapy from 2016 till 2019. Eligible patients were aged 18 to 75, with morphologically confirmed diagnosis of colon adenocarcinoma, Eastern Cooperative Oncology Group (ECOG) 0-1 with no major comorbidities.

\subsection{Treatment}

The study includes patients with newly diagnosed metastatic colorectal cancer and also the patients with disease recurrence and distant metastases after radical treatment. Prior adjuvant chemotherapy for CRC was allowed at least 6 months before inclusion. First-line induction treatment was given with FOLFOX/XELOX. After induction patients without progressive disease were eligible for maintenance treatment. They received metronomic regimen consisting of oral capecitabine $500 \mathrm{mg} 3$ times a day and oral cyclophosphane $50 \mathrm{mg}$ once a day. Treatment was given until disease progression, intolerable toxicity or death from any reasons. In case of capecitabine-related toxicity grade $\geq 2$, maintenance therapy was interrupted until toxicity resolved to grade $\leq 1$, then the treatment continued with capecitabine dose $500 \mathrm{mg}$ twice daily. Before the start of maintenance treatment liver biopsy was performed and blood samples were obtained for molecular studies. To investigate the potential biomarkers of sensitivity to metronomic chemotherapy we studied the expression of genes VEGFR1, VEGFR2, DPD, TP and TS in the fresh frozen tissue of liver metastases and VEGF level in blood serum.

The control arm included mCRC patients who received the same induction treatment without supportive chemotherapy from 2011 till 2015. The data of these patients were obtained from the retrospective analysis of National Cancer Registry and medical records.

\subsection{Biomarker Level Measurement}

The levels of CEA and CA 19-9 were determined in venous blood by the method of immunoferment analysis. To determine the level of VEGF protein in the blood serum of patients, the diagnostic reagent kit Milliplex Human MAP Human Angiogenesis/Growth Factor Magnetic Bead Panel 1 (Merck Millipore, USA) was used. To determine the expression profile of genes VEGFR1, VEGFR2, DPD, TP and TS in fresh frozen tissue of metastases of CRC, the method of real-time polymerase chain reaction (PCR) was used.

\subsection{Efficacy and Safety Evaluation}

The aim of the study was to evaluate whether maintenance treatment increases the progression-free survival (PFS) and overall survival (OS) compared with induction treatment alone. PFS and OS were counted from the start date of first-line chemotherapy. For survival analyses Kaplan-Meier method was used. For tumor response evaluation computed tomography (CT) scan was performed before the start of maintenance treatment and every 3 months during metronomic therapy. Response was evaluated according to RECIST 1.1 (Response Evaluation Criteria In Solid Tumors). A comparison of survival rates depending on various factors in groups was performed with the log-rank test. Multivariate statistical analysis of the influence of variables on the survival rate of patients was conducted by the Cox regression model. Toxic effects were recorded according to National Cancer Institute Common Terminology Criteria for Adverse Events NCI CTCAE (version 4.0). 


\section{Results and Discussion}

\subsection{Efficacy and Survival}

The study enrolled 140 patients, 70 in maintenance and 70 in control arms. To date, tumor response was evaluated in 60 patients. As the best response to treatment, 10 (16.7\%) patients had partial response (PR) and 29 (48.3\%) had stable disease (SD) for 3 months and more without intolerable toxicity. So, disease control was achieved in 39 cases $(65 \%)$.

To date, tumor progression was registered in 48 patients, the time from the start of induction chemotherapy to disease progression varied from 5.8 to 20.6 months. Patients received metronomic chemotherapy over a period of 2.3 to 12.0 months, the median duration of maintenance treatment was 6.3 months.

Median PFS from the start of first-line chemotherapy was 9.0 months and 7.4 months in the maintenance and control arms, respectively (Fig. 1).

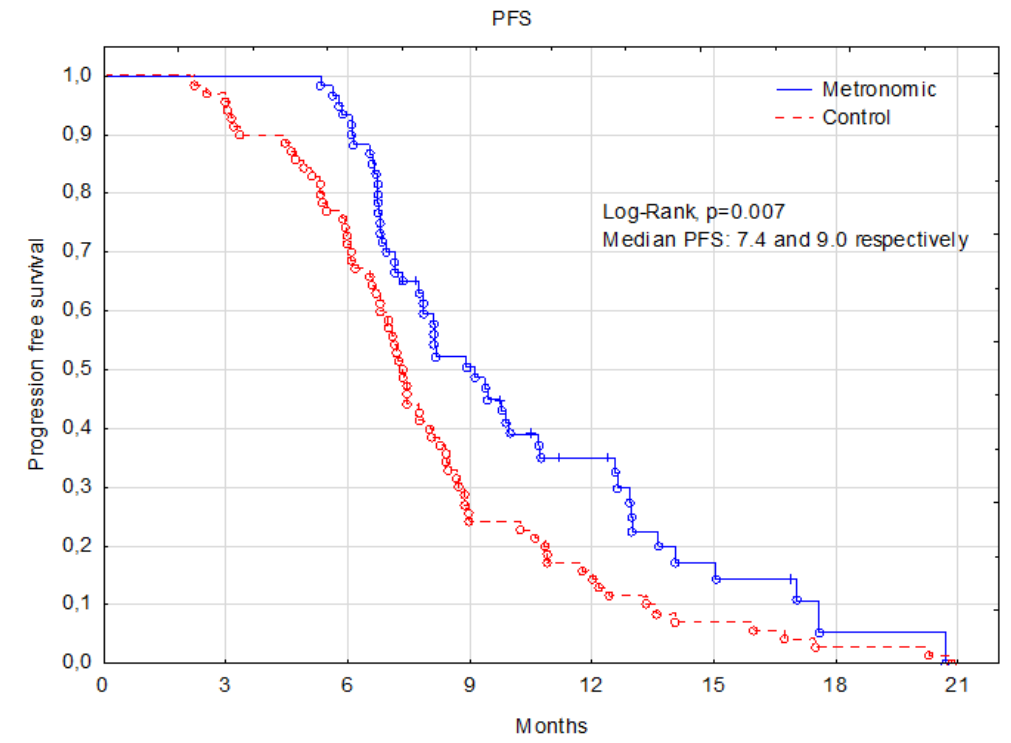

Figure 1. Progression-free survival of patients in the maintenance and control arms

At present moment, all patients in control arm and 32 patients in maintenance arm had died. Median OS in the group of patients on metronomic chemotherapy is 22.9 months, which far exceeds the corresponding rate in the control arm (14.7 months) (Fig. 2).

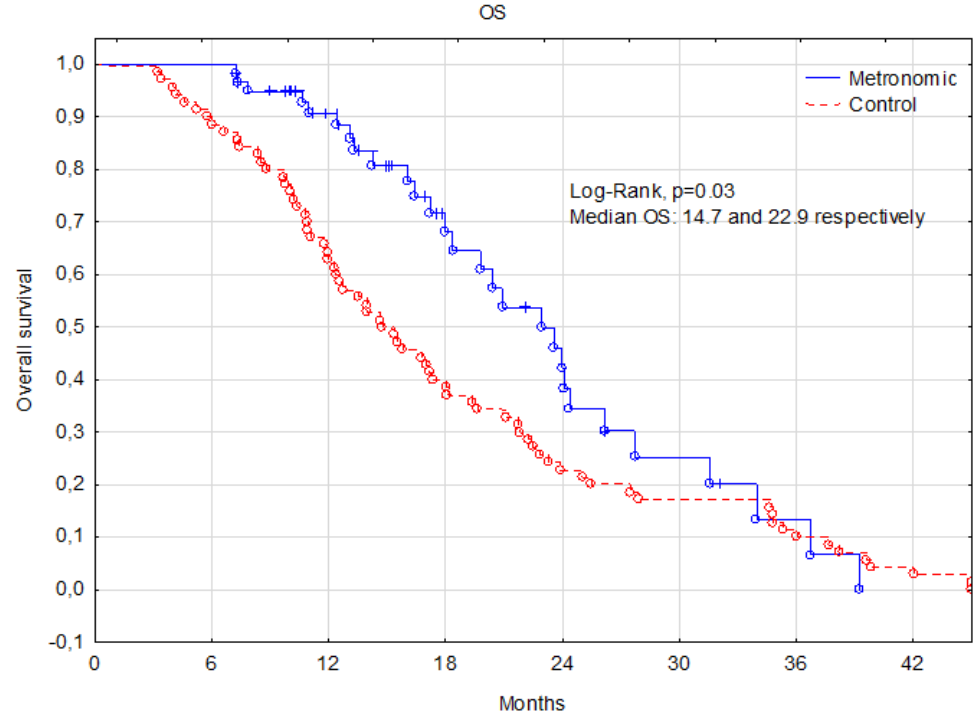

Figure 2. Overall survival of patients in the maintenance and control arms 


\subsection{Prognostic Factors for Metronomic Chemotherapy}

To determine clinical and laboratory prognostic factors affecting PFS, the Cox proportional hazards regression was made. As possible prognostic factors the patients sex, age (up to 60 years and older), tumor markers CEA and CA 19-9 levels of were selected.

Table 1. Regression analysis data of clinical and laboratory factors and their influence on the treatment efficacy

\begin{tabular}{lll}
\hline Test parameter & Regression coefficient & Difference significance, $\mathrm{p}$ \\
\hline Age & 0.87 & 0.38 \\
CEA & 1.67 & 0.09 \\
CA 19-9 & 1.83 & 0.07 \\
Sex & -0.26 & 0.8 \\
\hline
\end{tabular}

It was found that the CEA level of more than $10 \mathrm{ng} / \mathrm{ml}$ and the CA $19-9$ level of more than $70 \mathrm{ng} / \mathrm{ml}$ are reliably associated with a higher probability of adverse outcome ( $\mathrm{p}=0.006$ and $\mathrm{p}=0.005$ respectively) (Figs. 3, 4).

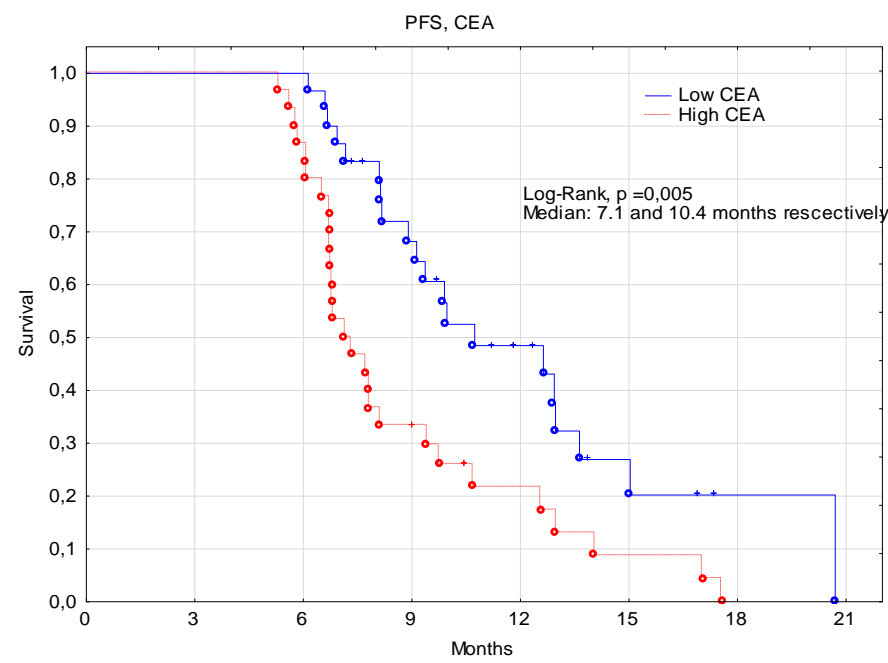

Figure 3. Progression-free survival in the maintenance arm depending on CEA level

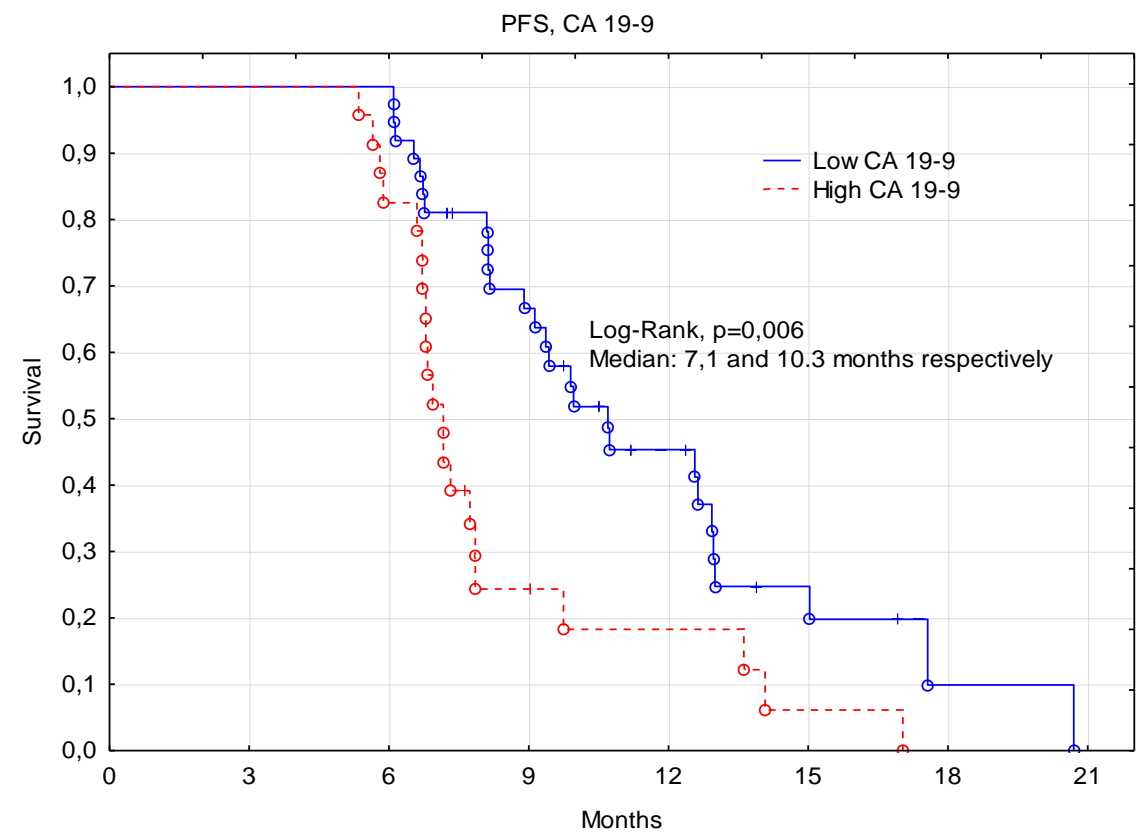

Figure 4. Progression-free survival in the maintenance arm depending CA 19-9 level 
To define the influence of molecular genetic factors on the efficacy of metronomic chemotherapy, the VEFG protein levels in the blood serum and the expression of the genes VEGFR1, VEGFR2, DPD, TP and TS were examined. In all, 40 blood serum samples were tested till present moment. For each serum marker (VEGF-A, VEGF-C, VEGF-D), the level of marker concentration was calculated in dynamics on metronomic chemotherapy over a period of the first two months from the start of treatment. The level differences in the groups of patients with positive effect (partial response and stable disease) and disease progression were compared (Fig. 5).
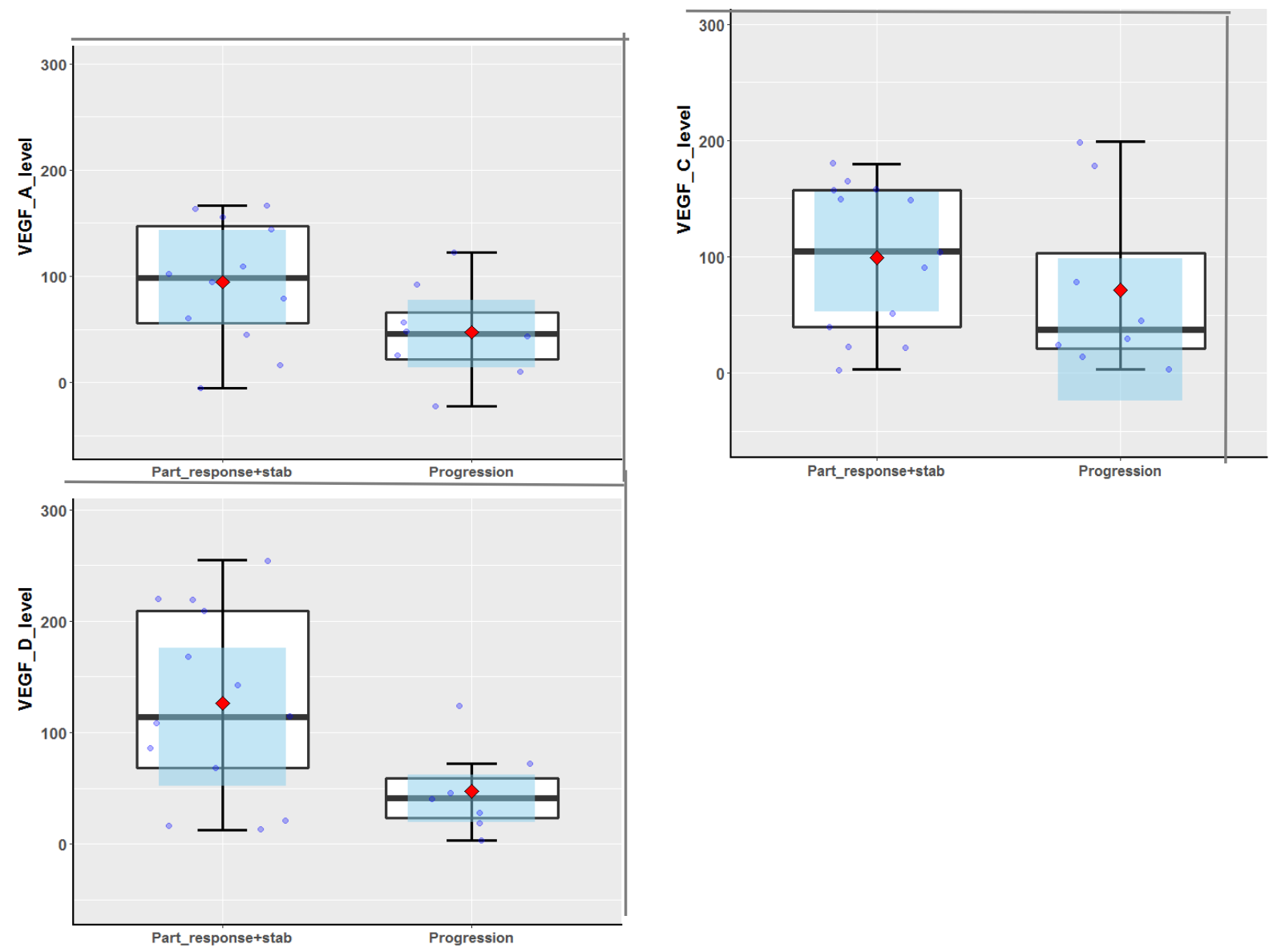

Figure 5. Dynamics of reduction of growth factors in groups with positive effect and with disease progression on metronomic therapy

The presented plots show that the concentration of growth factors in dynamics decreases slower in the group of patients with disease progression and can be a marker of the metronomic therapy efficacy; however the differences are not significant ( $\mathrm{p}=0.3, \mathrm{p}=0.68$ and $\mathrm{p}=0.082$ for VEGF-A, VEGF-C and VEGF-D respectively). This fact can be due to a limited number of studied samples and will be specified during further research.

The expression of the genes VEGFR1, VEGFR2, DPD, TP and TS was examined in 40 tumor tissue samples. To define the influence of the considered factors on the metronomic therapy efficacy in terms of PFS, the Cox proportional hazards regression was made. The obtained data show that the increase in the expression of TP gene is reliably associated with a higher probability of adverse outcome (disease progression) $(\mathrm{p}=0.0015)$ and, vice versa, the increase in the levels of TS, DPD and VEGFR1 genes - with a less probability of tumor progression $(\mathrm{p}=0.0201, \mathrm{p}=0.0200$ and $\mathrm{p}=0.0264$ respectively). For the expression of VEGFR2 gene a significant influence on the clinical outcome was not revealed (Table 2). 
Table 2. Regression analysis data of molecular genetic factors

\begin{tabular}{lll}
\hline Test gene & Regression coefficient & Difference significance, $\mathrm{p}$ \\
\hline VEGFR1 & 1.76 & 0.027 \\
VEGFR2 & -1.19 & 0.206 \\
DPD & 1.12 & 0.020 \\
TP & -2.80 & 0.002 \\
TS & 0.83 & 0.020 \\
\hline
\end{tabular}

Treatment was well tolerated. During the maintenance period 32 cases of different adverse events were recorded, the majority of them were grade 1 . Treatment of 7 patients with grade $2 / 3$ hematologic and skin toxicity was interrupted until toxicity resolved to grade $\leq 1$, then started again with a lower dose of capecitabine. No patients were excluded from the study because of adverse events.

\subsection{Discussion}

Our study is still to be continued, so the results presented here are not final and may change when efficacy evaluation and molecular studies results will be available for all patients enrolled. However, preliminary results clearly show the benefit of maintenance therapy, at least in group of patients with positive predictive factors. These data coincide with the results of many international clinical trials. OPTIMOX2 was the first study, which compared the fluoropyrimidine-based maintenance strategy with an intermittent approach and evaluated whether maintenance could be replaced by a "chemotherapy-free interval" after FOLFOX induction. It showed that patients who had received maintenance therapy had a significantly longer median duration of disease control (13.1 vs. 9.3 months), longer median PFS (8.6 vs. 6.6 months), and longer median OS (23.8 vs. 19.5 months) compared to those who discontinued treatment (Chibaudel et al., 2012). Later several trials including bevacizumab as maintenance treatment were inducted, in some of them, as in CAIRO3, it was given together with metronomic capecitabine. This strategy showed positive results, PFS was longer in the maintenance arm (11.7 vs. 8.5 months) (Koopman, Simkens, \& Tije, 2013). It gave us an idea of some benefit from antiangiogenic maintenance treatment for mCRC patients. As targeted agents are not always available in low and middle income countries because of their high price, metronomic regimen seemed to be a good alternative. Several preclinical studies on human CRC xenografts have demonstrated that low-dose cyclophosphamide, tegafur uracil, capecitabine and topotecan may all have antitumor activity (Bocci, Nicolau, \& Kerbel, 2002; Louvet, Coudray, \& Tournigand, 2000; Tsujimoto, Tsukioka, \& Ono, 2010). Then some clinical trials on small groups of patients have proved the efficacy of metronomic regimens. Interesting results were shown by Nordic ACT2 trial, in which patients with mutated KRAS were randomized to receive maintenance bevacizumab or metronomic capecitabine. The OS was longer in capecitabine, than in bevazizumab arm (28.0 and 26.4 months respectively) (Hagman et al., 2016). These results should be interpreted with caution due to small sample size, but still non-inferiority of metronomic regimen seems to be very promising for future studies.

It is now quite clear that different metronomic chemotherapy regimens can be effective in certain groups of patients. Therefore, the issue of searching for markers of sensitivity to such types of treatment is particularly relevant. For the time being, maintenance therapy should be considered a valuable option for selected patients rather than a standard of care. It remains unclear, however, which maintenance regimen is preferable for use and how to select the group of patients who may benefit the most from such treatment.

\section{Conclusion}

According to the preliminary data, metronomic maintenance chemotherapy allows to improve the efficacy of $\mathrm{mCRC}$ treatment without negative influence on the quality of life. Although some international trials demonstrate better survival rates, metronomic therapy is economically beneficial and can find use in middle and low income countries where the possibility to use targeted agents is limited. One of the main advantages of this method is its low toxicity, which reduces the need for hospitalization and supportive treatment (antiemetics, colony-stimulating factors, hemotransfusions).

The high expression levels of the genes TS, DPD and VEGFR1, the low expression level of the gene TP, as well as low levels of CEA and CA 19-9 tumor markers are the prognostic factors of sensitivity to metronomic chemotherapy given to mCRC patients. Based on these data, it is possible to identify a group of patients who can benefit from this method of treatment.

Since our research is still ongoing, we hope to identify a group of patients sensitive to low doses of cyclophosphamide and capecitabine more accurately. It remains unclear whether the level of VEGF is a reliable 
marker of sensitivity to such treatment. We plan to analyze the impact of all biomarkers on the treatment results in a larger group of patients.

\section{References}

Allegrini, G., Di Desidero, T., \& Barletta, M. (2012). Clinical, pharmacokinetic and pharmacodynamic evaluations of metronomic UFT and cyclophosphamide plus celecoxib in patients with advanced refractory gastrointestinal cancers. Angiogenesis, 15(2), 275-286. https://doi/10.1007/s10456-012-9260-6

Bocci, G., Nicolau, K. C., \& Kerbel, R. S. (2002, December 1). Protracted low-dose effects on human endothelial cell proliferation and survival in vitro reveal a selective antiangiogenic window for various chemotherapeutic drugs. Cancer Research, 62, 6938-6943.

Browder, T., Butterfield, C. E., \& Kraling, B. M. (2000, April 1). Antiangiogenic scheduling of chemotherapy improves efficacy against experimental drug-resistant cancer. Cancer Research, 60(7), 1878-1886.

Calleri, A., Bono, A., Bagnardi, V., \& Quarna, J. (2009, December 15). Predictive potential of angiogenic growth factors and circulating endothelial cells in breast cancer patients receiving metronomic chemotherapy plus bevacizumab. Clinical Cancer Research, 15(24), 7652-7765. https://doi.org/10.1158/1078-0432.CCR-09-1493

Chibaudel, B., Maindrault-Goebel, F., Lledo, G., Mineur, L., André, T., Bennamoun, M., \& de Gramont, A. (2009). Can chemotherapy be discontinued in unresectable metastatic colorectal cancer? The GERCOR OPTIMOX2 Study. Journal of Clinical Oncology, 27(34), 5727-5733. https://doi.org/10.1200/JCO.2009.23.4344

Chubenko, V. (2007, January 15). Perspective methods of treatment of malignant tumors. Practical oncology, 4, 228-234.

Drevs, J., Fakler, J., \& Eisele, S. (2004, June 1). Antiangiogenic potency of various chemotherapeutic drugs for metronomic chemotherapy. Anticancer Res, 24, 1759-1763.

Fedyanin, M. (2012, February 20). Personilized therapy in oncology: present and future. Malignant tumors, 2, 106-110.

Ferlay, J., Steliarova-Foucher, E., \& Lortet-Tieulent, J. (2013). Cancer incidence and mortality patterns in Europe: estimates for 40 countries in 2012. European Journal of Cancer, 49, 1374-1403. https://doi.org/10.1016/j.ejca.2012.12.027

Gnoni, A., Silvestris, N., \& Licchetta, A. (2015). Metronomic chemotherapy from rationale to clinical studies: a dream or reality? Critical Review Oncology Hematology, 95(1), 46-61. https://doi.org/10.1016/j.critrevonc.2015.01.008

Hagman, H., Frödin, J., Berglund, Å., Sundberg, J., Vestermark, L., Albertsson, M., .... Fernebro, E. (2016, January 1). A randomized study of KRAS-guided maintenance therapy with bevacizumab, erlotinib or metronomic capecitabine after first-line induction treatment of metastatic colorectal cancer: the Nordic ACT2 trial. Annals of Oncology, 27(1), 140-147. https://doi.org/10.1093/annonc/mdv490

Kerbel, R. S. (1991). Inhibition of tumor angiogenesis as a strategy to circumvent acquired resistance to anti-cancer therapeutic agents. Bioessays, 13(1). https://doi.org/10.1002/bies.950130106

Koopman, M., Simkens, L. H., \& Tije, A. J. (2014, May 20). Maintenance treatment with capecitabine and bevacizumab versus observation after induction treatment with chemotherapy and bevacizumab in metastatic colorectal cancer (mCRC): The phase III CAIRO3 study of the Dutch Colorectal Cancer Group (DCCG). Journal of Clinical Oncology, 32, 3504-3504. https://doi.org/10.1200/jco.2014.32.15_suppl.3504

Lien, K., Georgsdottir, S., Sivanathan, L., Chan, K., \& Emmenegger U. (2013). Low-dose metronomic chemotherapy: a systematic literature analysis. European Journal of Cancer, 49(16), 3387-3395. https://doi.org/10.1016/j.ejca.2013.06.038

Longley, D. B., Harkin, D. P., \& Johnston, P. G. (2003). 5-fluorouracil: mechanisms of action and clinical strategies. Nature Reviews Cancer, 3(5), 330-338. https://doi.org/10.1038/nrc1074

Louvet, C., Coudray, A. M., \& Tournigand, C. (2000, August 1). Synergistic antitumoral activity of combined UFT, folinic acid and oxaliplatin against human colorectal HT29 cell xenografts in athymic nude mice. Anticancer Drugs, 11, 579-582. https://doi.org/10.1097/00001813-200008000-00010

Miger, J., Holmqvist, A., Sun, X. F., \& Albertsson, M. (2014). Low-dose capecitabine (Xeloda) for treatment for 
gastrointestinal cancer. Medical Oncology, 31, 870. https://doi.org/10.1007/s12032-014-0870-2

Okeanov, A. E., Moiseyev, P. I., \& Levin, L. F. (2014). Statistics of cancer diseases in the Republic of Belarus (2004-2013). Edited by Sukonko OG. Minsk: N.N.Alexandrov National Cancer Centre of Belarus, 69-75.

Pasquier, E., Kavallaris, M., \& André, N. (2010). Metronomic chemotherapy: new rationale for new directions. Nature Reviews Clinical Oncology, 7(8), 455-465. https://doi.org/10.1038/nrclinonc.2010.82

Stöhlmacher, J. (2005). Pharmacogenetics in gastrointestinal tumors. Onkologie, 28, 435-440. https://doi.org/10.1159/000086499

Tsujimoto, H., Tsukioka, S., \& Ono, S. (2010, September 19). Effect of leucovorin on the antitumor efficacy of the 5-FU prodrug, tegafureuracil, in human colorectal cancer xenografts with various expression levels of thymidylate synthase. Oncology Letters, 1, 973-980. https://doi.org/10.3892/ol.2010.172

\section{Copyrights}

Copyright for this article is retained by the author(s), with first publication rights granted to the journal.

This is an open-access article distributed under the terms and conditions of the Creative Commons Attribution license (http://creativecommons.org/licenses/by/4.0/). 